\title{
Knowledge, Attitudes, and Clinical Practices of Dental Professionals during COVID-19 Pandemic in Pakistan
}

\author{
Khalid Almas ${ }^{1}$ Abdul Samad Khan 20 \\ Ashar Afaq ${ }^{3}$ Abdul Majeed ${ }^{4}$ \\ ${ }^{1}$ Department of Preventive Dental Sciences, College of Dentistry, \\ Imam Abdulrahman Bin Faisal University, Dammam, Saudi Arabia \\ ${ }^{2}$ Department of Restorative Dental Sciences, College of Dentistry, \\ Imam Abdulrahman Bin Faisal University, Dammam, Saudi Arabia \\ ${ }^{3}$ Department of Community Dentistry, Dow International Dental \\ College, Karachi, Pakistan \\ ${ }^{4}$ School of Dental Medicine, University of Colorado, Anschutz \\ Medical Campus, Aurora, USA
}

Eur J Dent:2020;14(suppl S1):S63-S69
Address for correspondence Muhammad Ashraf Nazir, BSc, BDS, MPH, FRSPH, Department of Preventive Dental Sciences, College of Dentistry, Imam Abdulrahman Bin Faisal University, P.O. Box 1982, Dammam 31441, Saudi Arabia (e-mail: manazir@iau.edu.sa).

\begin{abstract}
Keywords

- COVID-19

- pandemic

- dental professionals

- continuing education

- awareness

Objective The aim of this study is to assess knowledge, attitudes, and clinical practices of dental professionals regarding the prevention and control of coronavirus disease 2019 (COVID-19) in Pakistan.

Materials and Methods General dentists and dental specialists working in public and private dental practices, hospitals, and academic institutions participated in this cross-sectional study. A pilot-tested questionnaire was sent to dental professionals through an online link in Pakistan and data collection was completed in April-May 2020. The knowledge score was calculated from 22 variables about the COVID-19.

Results The study included data of 343 dental professionals with $47.2 \%$ of males and $52.8 \%$ of females. The mean knowledge score was $16.78 \pm 2.25$, and it significantly differed between general dentists $(16.55 \pm 2.36)$ and dental specialists $(17.15 \pm 2.04)(p=0.020)$, and those with up to 10 years of experience $(16.58 \pm 2.28)$ and those with more than 10 years of experience $(17.05 \pm 2.2)(p=0.026)$. Only $15.5 \%$ of the participants were comfortable in treating patients during the COVID-19 pandemic. A workshop/seminar on the COVID-19 was attended by $23 \%$ of the participants. In multivariate analysis, being comfortable in treating patients (odds ratio $=3.31,95 \%$ confidence interval $=1.63,6.73$ ) was associated with the attendance of workshop/seminar on COVID-19.

Conclusions Dental professionals had adequate knowledge about COVID-19, but a few of them were comfortable in treating patients during the pandemic. A minority of dental professionals attended a workshop/seminar on the COVID-19. Continuous education activities should be provided to dental professionals to enhance their role in the prevention of COVID-19 spread and promotion of oral health.
\end{abstract}

DOI https://doi.org/

$10.1055 / \mathrm{s}-0040-1718785$

ISSN 1305-7456.
(C) 2020. European Journal of Dentistry.

This is an open access article published by Thieme under the terms of the Creative Commons Attribution-NonDerivative-NonCommercial-License, permitting copying and reproduction so long as the original work is given appropriate credit. Contents may not be used for commercial purposes, or adapted, remixed, transformed or built upon. (https:// creativecommons.org/licenses/by-nc-nd/4.0/)

Thieme Medical and Scientific Publishers Pvt. Ltd. A-12, 2nd Floor, Sector 2, Noida-201301 UP, India 


\section{Introduction}

The novel coronavirus disease 2019 (COVID-19) is a newly discovered viral infection that started in Wuhan, China in December 2019 and caused the outbreak of pneumonia in the rest of the world. ${ }^{1}$ The World Health Organization (WHO) declared a public health emergency of international concern over this global outbreak on January 30, 2020. ${ }^{1}$ On February 11, 2020, the WHO named the novel viral pneumonia as "coronavirus disease (COVID-19)."2 On March 11, 2020, the WHO declared it as a controllable pandemic disease. ${ }^{3}$

As per the WHO, till May 27, 2020, there were 5.49 million confirmed positive cases worldwide with 0.349 million global death toll. ${ }^{4}$ New cases are reported every day and are increasing in an exponential manner. It has been spread in 217 countries, areas, and territories. Among these, four countries including Turkey, India, Saudi Arabia, and Pakistan are in top 20 highly reported countries. It is anticipated that COVID-19 is a disaster for developing nations not only medically but also economically and psychologically. The data recording these cases can be tracked on WHO Web site and many other platforms. ${ }^{4}$

Currently, there are four genera of coronaviruses: $\alpha-\mathrm{CoV}$, $\beta-\mathrm{CoV}, \gamma-\mathrm{CoV}$, and $\delta-\mathrm{CoV}^{5,6}$ Latest COVID-19 has caused severe respiratory infection which leads to severe pneumonia and eventually death in elderly population and immunocompromised individuals. ${ }^{1}$ The typical clinical symptoms of the patients who suffer from the COVID-19 are fever, cough, and myalgia or fatigue with abnormal chest CT scan, and less common symptoms are sputum production, headache, hemoptysis, and diarrhea., ${ }^{7,8}$

The common transmission routes of COVID-19 include direct (cough, sneeze, and droplet inhalation transmission) and contact (contact with oral, nasal, and eye mucous membranes) transmissions. ${ }^{9}$ Although common clinical manifestations of novel coronavirus infection do not include eye symptoms, the analysis of conjunctival samples from confirmed and suspected cases of 2019-nCoV or novel coronavirus suggested that eye exposure might provide an effective way for the virus to enter the body. ${ }^{10,11}$ Indirect spread of coronavirus has also been seen through the touch or close contact of the contaminated surfaces. ${ }^{1,11}$

Health care professionals including dentists are performing their duties in close contact with patients and they are at high risk of exposure to aerosol and droplets splashing out of patients' oral cavity. ${ }^{12}$ Therefore, all dental health care professionals should follow the latest infection control guidelines published by Disease Control and Prevention (CDC), ${ }^{13}$ the American Dental Association (ADA), ${ }^{14}$ and the $\mathrm{WHO}^{4}$ to prevent the spread of COVID-infection. The main recommendations of these guidelines are related to personal protective equipment and routes of transmission. ${ }^{1,11}$ It is critical for the dental professional to have optimal knowledge of prevention practices, that's how they can play their role in disease prevention and control. ${ }^{1,13,14}$ Therefore, the aim of the present study was to assess the knowledge, attitudes, and clinical practices of dental professionals regarding the prevention and control of COVID-19 disease during the pandemic.

\section{Materials and Methods}

The study population for this cross-sectional survey included dental professionals (general dentists and dental specialists) from Pakistan working in private dental practices, dental hospitals, and/or teaching institutions. A sample of 381 was calculated assuming 95\% confidence interval (CI), 5\% margin of error, population size $(N=22,000)$, and $50 \%$ response distribution. A convenience sample of dentists who wanted to voluntarily participate in the study was used. The study approval was obtained from the Institutional Review Board of the Dow University of Health Sciences, Karachi, Pakistan. The study was conducted as per the ethical guidelines of the Helsinki Declaration. ${ }^{15}$

Based on recent guidelines of $\mathrm{ADA},{ }^{14} \mathrm{WHO},{ }^{4} \mathrm{CDC},{ }^{13}$ and recent published literature, ${ }^{1,11,16}$ a questionnaire was developed and discussed among researchers (K.A., A.S.K., A.T., M.A.N.) to confirm its content validity. The content of each item in the questionnaire was carefully reviewed and discussed so that the questionnaire accurately assesses the aspect of COVID-19 research on dental professionals. The draft of the questionnaire was pretested among 20 dental professionals to ensure its reliability and validity. The pretested data were not included in the final results of the study. The final questionnaire was then created/developed at www.google. com/forms for distribution among study participants.

The questionnaire was in English language and consisted of two sections. Section one included questions regarding demographic data of study participants (age, gender, designation, years in practice, workplace, income, etc.). Section two consisted of series of multiple-choice questions related to the awareness, knowledge, and clinical practices of dental professionals with respect to COVID-19 signs and symptoms, routes of transmission, patient evaluation, treatment, and infection control protocol. In addition to these, there were questions about preparedness, willingness to treat patients, impacts of COVID-19, attendance of workshop/seminar on COVID-19, and willingness to know more about the disease.

The survey was conducted in April-May 2020 through an online questionnaire. The questionnaire was circulated through social media outlets (WhatsApp, Facebook) and online submissions through emails were received during this period. Participants could fill the questionnaire only once to avoid duplication. Incomplete questionnaire feedback was not included in the study and all received data were anonymous and confidential.

\section{Variables Description}

There were 22 variables regarding knowledge/awareness of dental professionals about COVID-19. These variables broadly assessed infection prevention measures, patient screening including COVID-19 signs and symptoms, and patient management. Five variables such as being comfortable in treating patients, refusing patients diagnosed with COVID-19, maintaining clinical protocols, attending seminars/workshops, and receiving more information, were used to evaluate the attitudes/clinical practice of dentists. 
The independent variables of the study included gender, place of work, monthly income, job status, experience, qualifications obtained from the public or private institute, comfortability in treating patients, refusing patients, following clinical protocols, and willingness to receive more information. These variables were used to assess their association with the attendance of workshop/seminar on COVID-19 (dependent variable).

\section{Data Analysis}

The data were analyzed using the Statistical Package for Social Sciences (SPSS) version 22 (IBM Corp.; Armonk, New York, United States). Descriptive statistics were used to describe items included in the survey. Means and standard deviations were used to describe the continuous variables and frequency and percentages were used to describe the categorical data. The knowledge score of each participant was calculated by adding the scores of 22 variables. Mann-Whitney U-test was performed to compare knowledge score between different categories of participants (males vs. females, dentists vs. specialists, etc.) Bivariate and multiple logistic regression analyses were performed to evaluate the association of independent variables with the attendance of seminar/workshops. The significance level was set at $5 \%$.

\section{Results}

- Table 1 presents demographic information of the participants and comparison of their knowledge score during the COVID-19 pandemic. Of 381 dental professionals,
343 returned completed questionnaires and the response rate was $90 \%$ in the study. There were $47.2 \%$ of males and $52.8 \%$ of females in the study. More than half the participants were specialists (58.6\%), had a monthly income of more than 1,000 U.S. dollars (55.4\%), worked in the private sector (60.3\%), and had more than 10 years of experience (57.1\%). The mean knowledge score was $16.78 \pm 2.25$, and the minimum score was 6 and the maximum was 21. Mean knowledge score significantly differed between general dentists $(16.55 \pm 2.36)$ and dental specialists $(17.15 \pm 2.04)$ $(p=0.020)$, and those with up to 10 years of experience $(16.58 \pm 2.28)$ and those with more than 10 years of experience $(17.05 \pm 2.2)(p=0.026)$. The study found no significant difference in knowledge score with regards to gender, monthly income, place of work, and qualifications obtained from private and public institutions.

In response to a question about the action taken in the case of a patient with an elevated temperature without respiratory symptoms, $38.4 \%$ of participants reported sending the patient home and advising self-isolation. However, in the case of a patient with an elevated temperature with respiratory symptoms, $60.8 \%$ of participants reported to quarantine the patient and to report to the infection control department. Very few participants reported performing the required elective treatment on a patient with an elevated temperature and with or without respiratory symptoms ( - Fig. 1 ). The majority of participants $(86.7 \%$ ) reported to quarantine the patient and to report to the infection control department in case of a confirmed COVID19 case (-Fig. 2 ).

Table 1 Comparison of knowledge scores in different categories of participants

\begin{tabular}{|c|c|c|c|}
\hline Variables & Frequency (\%) & $\begin{array}{l}\text { Knowledge score } \\
\text { mean (SD) }\end{array}$ & $p$-Value \\
\hline \multicolumn{3}{|l|}{ Gender } & \multirow[t]{3}{*}{0.358} \\
\hline Male & $162(47.2)$ & $16.79 \pm 2.42$ & \\
\hline Female & $181(52.8)$ & $16.77 \pm 2.11$ & \\
\hline \multicolumn{3}{|l|}{ Monthly income } & \multirow[t]{3}{*}{0.269} \\
\hline Up to 1,000 U.S. dollars & $153(44.6)$ & $16.6 \pm 2.47$ & \\
\hline More than 1,000 U.S. dollars & $190(55.4)$ & $16.93 \pm 2.06$ & \\
\hline \multicolumn{3}{|l|}{ Place of work } & \multirow[t]{3}{*}{0.496} \\
\hline Government sector & $136(39.7)$ & $16.74 \pm 2.07$ & \\
\hline Private sector & $207(60.3)$ & $16.81 \pm 2.37$ & \\
\hline \multicolumn{3}{|l|}{ Qualification obtained } & \multirow[t]{3}{*}{0.212} \\
\hline Government institution & $212(61.8)$ & $16.94 \pm 2.27$ & \\
\hline Private institution & $131(38.2)$ & $16.67 \pm 2.24$ & \\
\hline \multicolumn{3}{|l|}{ Job status } & \multirow[t]{3}{*}{0.020} \\
\hline Dentist & $142(41.4)$ & $16.55 \pm 2.36$ & \\
\hline Dental Specialist & $201(58.6)$ & $17.15 \pm 2.04$ & \\
\hline \multicolumn{3}{|l|}{ Experience } & \multirow[t]{3}{*}{0.026} \\
\hline Up to $10 \mathrm{y}$ & $196(57.1)$ & $16.58 \pm 2.28$ & \\
\hline More than $10 \mathrm{y}$ & $147(42.9)$ & $17.05 \pm 2.2$ & \\
\hline
\end{tabular}

Abbreviation: SD, standard deviation. 


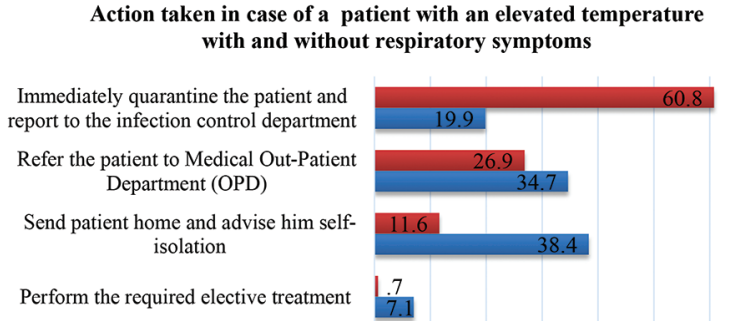

$\begin{array}{llllllll}0.0 & 10.0 & 20.0 & 30.0 & 40.0 & 50.0 & 60.0 & 70.0\end{array}$

- Elevated temperature with respiratory symptoms

- Elevated temperature without respiratory symptoms

Fig. 1 Distribution of participants' responses about the action taken in case of a patient with an elevated temperature but with and without the recent onset of respiratory symptoms.

Actions taken in case of confirmed COVID-19 patient

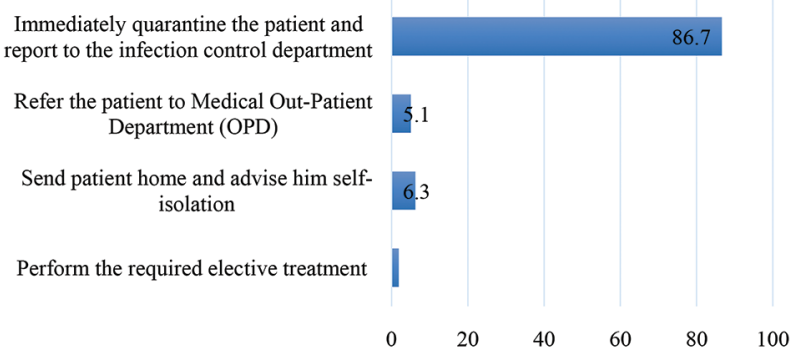

Fig. 2 Distribution of participants' responses about the actions taken in case of confirmed COVID-19 patient. COVID-19, coronavirus disease 2019.

Regarding dental procedures, $91.2 \%$ of participants mentioned to only perform emergency procedures, whereas $6.5 \%$ described not to perform any dental procedure during the COVID-19 pandemic ( - Fig. 3). Financial impact $(47.4 \%$ ) was one of the most commonly reported impacts of the COVID19 pandemic on the dental practice followed by psychological (33.8\%), social (14.9\%), and behavioral (3.9\%) impacts (-Fig. 4).

- Table 2 shows the attitudes of dentists during the COVID19 pandemic. Only $15.5 \%$ of participants were comfortable in treating patients during the COVID-19 pandemic. A workshop/ seminar on the COVID-19 pandemic was attended by $23 \%$ of the participants. Clinical protocols to deal with the COVID-19 pandemic were available with $54.8 \%$ of the participants.

In - Table 3, bivariate and multivariate analyses of data show the relationship of different factors with the attendance of workshop/seminar on COVID-19 pandemic. The bivariate analysis shows that being comfortable in treating patients (odds ratio $[\mathrm{OR}]=3.2,95 \% \mathrm{CI}=1.73,5.93$ ) and having clinical protocols ( $\mathrm{OR}=2.07,95 \% \mathrm{CI}=1.24,3.44)$ during the COVID-19 pandemic were significantly associated with increased odds of attending workshop/seminar. On the other hand, significantly reduced odds of attending workshop/seminar were associated with being a dentist $(\mathrm{OR}=0.45,95 \% \mathrm{CI}=0.27,0.75)$ and having a low monthly income ( $\mathrm{OR}=0.53,95 \% \mathrm{CI}=0.31,0.9$ ). In multivariate analysis, being comfortable in treating patients

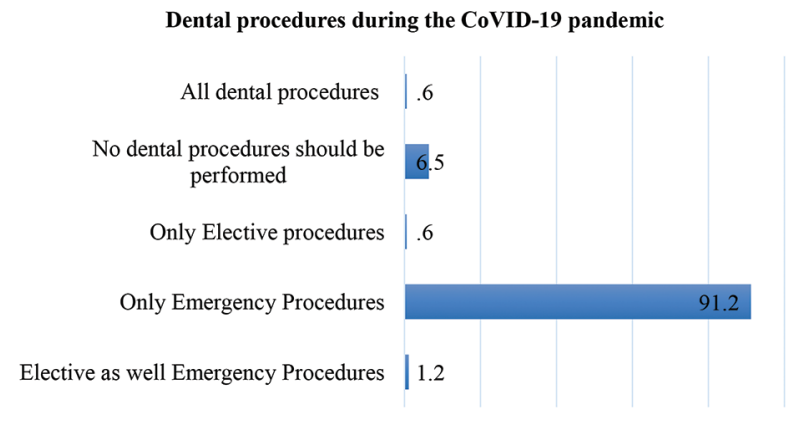

$\begin{array}{llllll}0.0 & 20.0 & 40.0 & 60.0 & 80.0 & 100.0\end{array}$

Fig. 3 Distribution of participants' responses about dental procedures during the COVID-19 pandemic. COVID-19, coronavirus disease 2019.

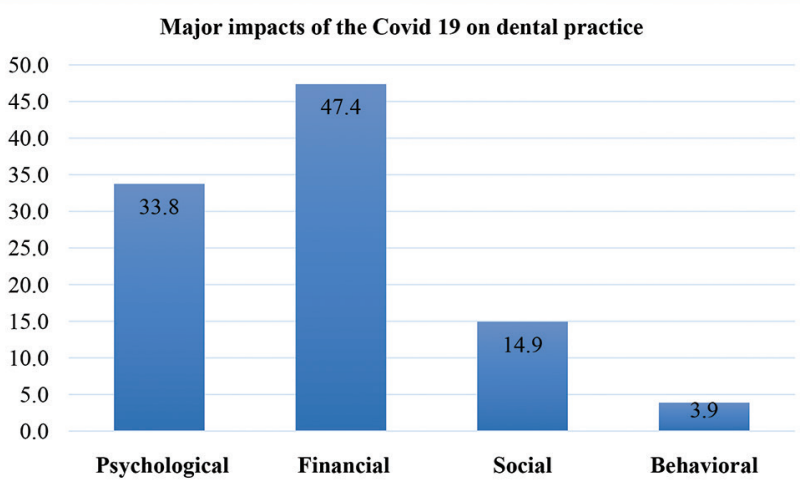

Fig. 4 Distribution of participants' responses about the major impacts of the COVID-19 pandemic on dental practice. COVID-19, coronavirus disease 2019.

Table 2 Participants' responses about clinical practice during the COVID-19 pandemic

\begin{tabular}{|l|l|}
\hline Variables & Frequency (\%) \\
\hline Comfortable in treating patients during the COVID-19 pandemic \\
\hline Yes & $53(15.5)$ \\
\hline No & $290(84.5)$ \\
\hline Refuse treating a patient with COVID-19 positive diagnosis \\
\hline Yes & $188(54.8)$ \\
\hline No & $155(45.2)$ \\
\hline Clinical protocols to deal with current COVID-19 pandemic \\
\hline Yes & $148(43.1)$ \\
\hline No & $195(56.9)$ \\
\hline Attend a workshop/seminar on COVID-19 pandemic \\
\hline Yes & $79(23.0)$ \\
\hline No & $264(77.0)$ \\
\hline Willing to receive information about COVID-19 pandemic \\
\hline Yes & $321(93.6)$ \\
\hline No & $22(6.4)$ \\
\hline
\end{tabular}

Abbreviation: COVID-19, coronavirus disease 2019.

$(\mathrm{OR}=3.31,95 \% \mathrm{CI}=1.63,6.73)$ and being a dentist $(\mathrm{OR}=0.49$ $95 \% \mathrm{CI}=0.27,0.86$ ) remained significant factors associated with the attendance of workshop/seminar. 
Table 3 Attendance of workshop: relationship with different factors

\begin{tabular}{|c|c|c|c|c|}
\hline Variables & $\begin{array}{l}\text { Unadjusted odds } \\
\text { ratio }\end{array}$ & p-Values & $\begin{array}{l}\text { Adjusted odds } \\
\text { ratio }\end{array}$ & $p$-Value \\
\hline $\begin{array}{l}\text { Gender } \\
\text { Male } \\
\text { Female }\end{array}$ & $1.27(0.77,2.11)$ & 0.343 & $1.07(0.62,1.86)$ & 0.798 \\
\hline $\begin{array}{l}\text { Monthly income } \\
\text { Up to } 1,000 \text { U.S. dollars (low income) } \\
\text { More than } 1,000 \text { U.S. dollars }\end{array}$ & $0.53(0.31,0.9)$ & 0.017 & $0.67(0.36,1.25)$ & 0.212 \\
\hline $\begin{array}{l}\text { Place of work } \\
\text { Government sector } \\
\text { Private sector }\end{array}$ & $1.05(0.63,1.75)$ & 0.859 & $1.08(0.61,1.92)$ & 0.781 \\
\hline $\begin{array}{l}\text { Qualification obtained } \\
\text { Government institution } \\
\text { Private institution }\end{array}$ & $1.25(0.75,2.07)$ & 0.391 & $1.09(0.61,1.94)$ & 0.778 \\
\hline $\begin{array}{l}\text { Current status } \\
\text { Dentist } \\
\text { Dental specialist }\end{array}$ & $0.45(0.27,0.75)$ & 0.002 & $0.49(0.27,0.86)$ & 0.014 \\
\hline $\begin{array}{l}\text { Experience } \\
\text { Up to } 10 \mathrm{y} \\
\text { More than } 10 \mathrm{y}\end{array}$ & $0.66(0.4,1.1)$ & 0.111 & $0.92(0.51,1.65)$ & 0.783 \\
\hline $\begin{array}{l}\text { Comfortable in treating patients during the } \\
\text { COVID-19 pandemic } \\
\text { Yes } \\
\text { No }\end{array}$ & $3.2(1.73,5.93)$ & $<0.001$ & $3.31(1.63,6.73)$ & 0.001 \\
\hline $\begin{array}{l}\text { Refuse treating a patient with } \\
\text { COVID-19 positive diagnosis } \\
\text { Yes } \\
\text { No }\end{array}$ & $0.75(0.46,1.25)$ & 0.268 & $1.19(0.66,2.14)$ & 0.554 \\
\hline $\begin{array}{l}\text { Clinical protocols to deal with current } \\
\text { COVID-19 pandemic } \\
\text { Yes } \\
\text { No }\end{array}$ & $2.07(1.24,3.44)$ & 0.005 & $1.61(0.93,2.79)$ & 0.090 \\
\hline $\begin{array}{l}\text { Willing to receive information about } \\
\text { COVID-19 pandemic } \\
\text { Yes } \\
\text { No }\end{array}$ & $0.62(0.24,1.58)$ & 0.312 & $0.79(0.29,2.15)$ & 0.640 \\
\hline
\end{tabular}

Abbreviation: COVID-19, coronavirus disease 2019.

\section{Discussion}

During the COVID-19 pandemic, all medical and dental professionals are confronting with new challenges in offering optimal treatment to their patients. ${ }^{17,18}$ By strict compliance to infection prevention and control policies, dental care providers can play a vital role in the flattening of the COVID-19 epidemic curve and preventing the complete failure of the health care system. Therefore, the present cross-sectional study provided an insight into knowledge, attitude, and practices of dental professionals during COVID-19 pandemic.

In the present study, dentists and dental specialists had optimal knowledge of modes of COVID-19 disease transmission, signs and symptoms of the disease, and mandatory preventive measures. Our findings were in accordance with a recently published study where the majority $(94.5 \%)$ of the health care professionals exhibited adequate awareness and knowledge regarding COVID-19. ${ }^{19}$ In another cross-sectional study, Khader et $\mathrm{al}^{20}$ reported similar results that Jordanian dentists had adequate knowledge related to COVID-19 mode of transmission, symptoms, infection control, and prevention in dental offices. On the other hand, a recent study by Sarfaraz et al indicated low knowledge about disinfection protocol during COVID-19 pandemic among dental practitioners and clinical post-graduate students globally. ${ }^{21}$

The mean knowledge score was significantly higher for dental specialists as compared with general dentists in the present study. In addition, the years of practical experience positively affected the knowledge score in our sample. In contrary to our findings, Ahmed et $\mathrm{a}^{19}$ observed no significant relationship $(p>0.05)$ between the health care providers' knowledge and their education level. The higher level of knowledge in specialists and experienced dental professionals is related to their training in the specialist program and greater learning in dental practice over years, respectively. The results of the present study exhibited no significant difference in knowledge score with regards to gender. A previous study of health care professionals in Pakistan reported no gender difference in their knowledge about COVID-19. ${ }^{19}$

In the present study, $38.4 \%$ of participants reported sending the patient home and advising self-isolation if the patient had elevated temperature without respiratory symptoms. However, $60.8 \%$ of participants agreed to quarantine the patient if he/she had an elevated body temperature along with 
respiratory symptoms. Our observations were in line with a recently published study in which $69.6 \%$ of the participants considered that the best management strategy for suspected COVID-19 individuals was self-isolation at home and seriously following social distancing measures. ${ }^{20}$ In the present study, $55 \%$ of the participants refused to provide any type of dental treatment to a patient with COVID-19 and only $15 \%$ were comfortable in providing dental treatments during the pandemic. The attitudes of dentists in the present study were similar to Jordanian dentists where the majority of participants (82.6\%) preferred to avoid treatment of a suspected COVID-19 patient even without signs and symptoms of the disease. ${ }^{20}$

In this study, $91.2 \%$ of participants reported providing only emergency dental care to their patients, whereas 6.5\% preferred not to perform any dental procedure during the COVID19 pandemic. These results show strict compliance of the study participants with CDC and ADA guidelines which recommended dentists to postpone all elective procedures and focus on only emergency dental care to reduce COVID-19 infection transmission. ${ }^{13,14}$ As it has been well-established that the main route for coronavirus transmission is via saliva and aerosols, this increases the probability of dentists and dental assistants from getting infected. ${ }^{1,22}$ In the current situation, all elective dental procedures should be postponed until the situation is under control. ${ }^{1,22}$ The literature also indicates marked decline in the number of patients reporting for emergency dental treatments during the COVID-19 pandemic. ${ }^{22}$

During the COVID-19 outbreak, dental health care professionals should take a comprehensive medical history, history of recent travel, or contact and measurement of body temperature for all patients presenting at dental offices as a routine procedure. ${ }^{23}$ Based on recent guidelines, dentists should take comprehensive personal protective measures and should avoid all the dental procedures that cause the production of droplets or aerosols. ${ }^{1,23}$ Four-handed dentistry, pretreatment mouthwash, and high-volume ejectors can significantly reduce aerosols production. ${ }^{11}$ The dentists should also encourage the testing of suspected cases of COVID-19 using saliva specimen, an approach which is novel, noninvasive, and nondistressing for patients. ${ }^{24}$ All dental practices and hospitals must follow comprehensive clinical protocols to prevent disease transmission during this pandemic. However, in the present study, only $54.8 \%$ of the participants reported that they had clinical protocols in their practices to deal with the challenges of the current COVID-19 pandemic.

The financial impact (47.4\%) was the most commonly reported impact of the COVID-19 pandemic on dental practice in our study. In private dental clinics, the provision of dental care only for emergency dental procedures will reduce the income of the clinics and contribute to financial stress. ${ }^{11}$ Globally, private health care is not mostly supported by the government, and therefore, it can cause a huge financial burden on dentists. This economic crisis has emphasized the significance of social security as well as the financial education of the dental team.

Psychological impacts in the form of fear and anxiety were quite prevalent in the current pandemic, particularly when the number of infected individuals is increasing every day and the mortality rate is rising exponentially. ${ }^{25}$ Several reports have emerged about the infection and deaths of health care providers from COIVD-19 infections. ${ }^{26}$ Moreover, there is no vaccine or appropriate treatment available, which increases stress and anxiety. ${ }^{27}$ These reasons may account for $34 \%$ of participants in the present study reporting the psychological impact of the COVID-19. Our results were in accordance with a recently performed study, where many dentists had reported fear of getting infected from their patients or coworkers during treatment procedures. ${ }^{25}$ Psychological trauma, fear of getting infected, or infecting a relative or family member had been reported among health care providers during previous similar outbreaks of other infections such as severe acute respiratory syndrome (SARS). ${ }^{28,29}$ Besides, health care providers carried the fear of being quarantined as a result of suspected COVID-19 patients and how their family members would cope during this stressful period. ${ }^{25}$ This additional financial burden in terms of cost of treatment further enhances one's stress. ${ }^{25}$ Therefore, psychological coping tools and strategies should be practiced among the dental community to stay calm and perform their duties professionally.

A workshop/seminar on the COVID-19 pandemic was attended by $23 \%$ of the participants. This was in accordance with a similar study amongst the health care professionals where 24.4.\% attended a professional scientific workshop/seminar related to COVID-19. ${ }^{19}$ On the contrary, Shi et al $^{30}$ reported that $64.63 \%$ of health care professionals attended a training program on COVID-19. In the present study, a positive relationship was observed between attending workshops/seminars and being comfortable in treating patients and having clinical protocols during the pandemic. Those who were comfortable in treating patients were 3.31 times more likely to attend the workshop than those who were not comfortable. Similarly, dentists were 0.49 times less likely to attend the workshop/seminar than specialists. Low attendance of workshops/seminars in our study can be related to the lack of continuing education opportunities in the country. There is a substantial need for online workshops, training, and seminars to significantly improve awareness, attitude, and practices of dental health care providers as $93.6 \%$ of our study participants were willing to receive information about COVID-19.

The limitation of the present study was the use of self-reported data that was subjected to under or overestimation of the responses by the study participants. Moreover, the data were collected in 2 months, and currently pandemic is spreading rapidly in Pakistan making dental health care providers more cautious regarding the COVID-19 disease transmission. Therefore, knowledge, attitudes, and practices of dental professionals might change with the emerging research and prospective treatment of COVID-19.

\section{Conclusions}

The study suggests that dentists and dental specialist participated in the study had adequate knowledge related to 
COVID-19. However, only half of them had clinical protocols in accordance with recent guidelines for their practices, and one-quarter attended COVID-19-related continuing education activities. Dental practitioners reported had huge financial and psychological impacts due to the COVID-19 pandemic.

\section{Recommendations}

- Continued professional development programs should be available and mandatory for all dental and other health care professionals to effectively prevent and control disease transmission, and possibly to reduce the related morbidity and mortality.

- General dentists and those dental professionals who are not comfortable in treating patients should be the main target of these continuing professional development activities.

- Financial as well as psychological support should be provided to all dental professionals who are putting their lives at risk for the wellbeing of their patients.

\section{Funding}

None.

\section{Conflict of Interest}

None declared.

\section{References}

1 Meng L, Hua F, Bian Z. Coronavirus disease 2019 (COVID-19): emerging and future challenges for dental and oral medicine. J Dent Res 2020;99(5):481-487

2 Gorbalenya AE, Baker SC, Baric R, Groot RJd, Drosten C, Gulyaeva AA, et al Severe acute respiratory syndrome-related coronavirus: The species and its viruses-a statement of the Coronavirus Study Group. Nat Microbiol 2020;5:536-544. doi:10.1101/2020.02.07.937862

3 Neher RA, Dyrdak R, Druelle V, Hodcroft EB, Albert J. Potential impact of seasonal forcing on a SARS-CoV-2 pandemic. Swiss Med Wkly 2020;150:w20224

4 World Health Organization, Clinical Management of Severe Acute Respiratory Infection When Novel Coronavirus (nCoV) Infection Is Suspected: Interim Guidance, 25 January 2020. World Health Organization; Geneva, Switzerland:2020

5 Nakagawa K, Lokugamage KG, Makino S. Viral and cellular mRNA translation in coronavirus-infected cells. Adv Virus Res 2016;96:165-192

6 Fan Y, Zhao K, Shi Z-L, Zhou P. Bat coronaviruses in China. Viruses 2019;11(3):210

7 Huang C, Wang Y, Li X, et al. Clinical features of patients infected with 2019 novel coronavirus in Wuhan, China. Lancet 2020;395(10223):497-506

8 Wang $\mathrm{D}, \mathrm{Hu} \mathrm{B}, \mathrm{Hu} \mathrm{C}$, et al. Clinical characteristics of 138 hospitalized patients with 2019 novel coronavirus-infected pneumonia in Wuhan, China. JAMA 2020;323:1061-1069

9 Lu CW, Liu XF, Jia ZF. 2019-nCoV transmission through the ocular surface must not be ignored. Lancet 2020;395(10224) :e39

10 To KK-W, Tsang OT-Y, Yip CC-Y, et al. Consistent detection of 2019 novel coronavirus in saliva. Clin Infect Dis 2020;71(15):841-843

11 Peng X, Xu X, Li Y, Cheng L, Zhou X, Ren B. Transmission routes of 2019-nCoV and controls in dental practice. Int J Oral Sci 2020;12(1):9

12 Ather A, Patel B, Ruparel NB, Diogenes A, Hargreaves KM. Coronavirus disease 19 (COVID-19): implications for clinical dental care. J Endod 2020;46(5):584-595
13 Centers for Disease Control and Prevention, CDC Developing Guidance Regarding Responding to COVID-19 in Dental Settings. Division of Oral Health. National Center for Chronic Disease Prevention and Health Promotion; Atlanta, GA, United States: 2020

14 American Dental Association. Coronavirus Frequently Asked Questions; 2020. Available at: https://success.ada.org/ en/practice-management/patients/coronavirus-frequently-asked-questions. Accessed June 20, 2020

15 General Assembly of the World Medical Association. World Medical Association Declaration of Helsinki: ethical principles for medical research involving human subjects. J Am Coll Dent 2014;81(3):14-18

16 Gaffar BO, El Tantawi M, Al-Ansari AA, AlAgl AS, Farooqi FA, Almas KM. Knowledge and practices of dentists regarding MERS-CoV. A cross-sectional survey in Saudi Arabia. Saudi Med J 2019;40(7):714-720

17 Cortegiani A, Ingoglia G, Ippolito M, Giarratano A, Einav S. A systematic review on the efficacy and safety of chloroquine for the treatment of COVID-19. J Crit Care 2020;57:279-283

18 van Doremalen N, Bushmaker T, Morris DH, et al. Aerosol and surface stability of SARS-CoV-2 as compared with SARS-CoV-1. N Engl J Med 2020;382(16):1564-1567

19 Ahmed N, Shakoor M, Vohra F, Abduljabbar T, Mariam Q Rehman MA. Knowledge, awareness and practice of health care professionals amid SARS-CoV-2, corona virus disease outbreak. Pak J Med Sci 2020;36(COVID19-S4) :S49-S56

20 Khader Y, Al Nsour M, Al-Batayneh OB, et al. Dentists' awareness, perception, and attitude regarding COVID-19 and infection control: cross-sectional study among Jordanian dentists. JMIR Public Health Surveill 2020;6(2):e18798

21 Sarfaraz S, Shabbir J, Mudasser MA, et al. Knowledge and attitude of dental practitioners related to disinfection during the COVID-19 pandemic. Healthcare (Basel) 2020;8(3):232

22 Guo H,Zhou Y, Liu X, Tan J. The impact of the COVID-19 epidemic on the utilization of emergency dental services. J Dent Sci 2020 (e-pub ahead of print). Doi: 10.1016/j.jds.2020.02.002

23 Guan WJ, Ni ZY, Hu Y, et al; China Medical Treatment Expert Group for Covid-19. Clinical characteristics of coronavirus disease 2019 in China. N Engl J Med 2020;382(18):1708-1720

24 Hamid H, Khurshid Z, Adanir N, Zafar MS, Zohaib S. COVID-19 pandemic and role of human saliva as a testing biofluid in point-of-care technology. Eur J Dent 2020;14(suppl S1): S123-S129 doi:10.1055/s-0040-1713020

25 Ahmed MA, Jouhar R, Ahmed N, et al. Fear and practice modifications among dentists to combat novel coronavirus disease (COVID-19) outbreak. Int J Environ Res Public Health 2020;17(8):2821

26 Kursumovic E, Lennane S, Cook TM. Deaths in healthcare workers due to COVID-19: the need for robust data and analysis. Anaesthesia 2020;75(8):989-992

27 Lai J, Ma S, Wang Y, et al. Factors associated with mental health outcomes among health care workers exposed to coronavirus disease 2019. JAMA Netw Open 2020;3(3):e203976

28 Tam CW, Pang EP, Lam LC, Chiu HF. Severe acute respiratory syndrome (SARS) in Hong Kong in 2003: stress and psychological impact among frontline healthcare workers. Psychol Med 2004;34(7):1197-1204

29 McAlonan GM, Lee AM, Cheung V, et al. Immediate and sustained psychological impact of an emerging infectious disease outbreak on health care workers. Can J Psychiatry 2007; 52(4):241-247

30 Shi Y, Wang J, Yang Y, et al. Knowledge and attitudes of medical staff in Chinese psychiatric hospitals regarding COVID-19. Brain Behav Immun Health 2020;4:100064 